OPEN ACCESS

Edited by:

Bang Shen,

Huazhong Agricultural University,

China

Reviewed by:

Haiqi He,

U.S. Department of Agriculture, USA

Anja Joachim,

Vetmeduni Vienna, Austria

Jianxun Luo,

Lanzhou Veterinary Research

Institute - Chinese Academy

of Agricultural Sciences, China

*Correspondence:

Quan Liu

liuquan1973@hotmail.com

Jun Qian

qianj1970@126.com

Specialty section:

This article was submitted to

Infectious Diseases,

a section of the journal

Frontiers in Microbiology

Received: 18 September 2016 Accepted: 15 November 2016 Published: 29 November 2016

Citation:

Wei F, Song $M$, Liu $H$, Wang $B$,

Wang S, Wang Z, Ma H, Li Z, Zeng Z, Qian J and Liu Q (2016)

Molecular Detection and Characterization of Zoonotic and Veterinary Pathogens in Ticks

from Northeastern China.

Front. Microbiol. 7:1913.

doi: 10.3389/fmicb.2016.01913

\section{Molecular Detection and Characterization of Zoonotic and Veterinary Pathogens in Ticks from Northeastern China}

Feng Wei ${ }^{1,2}$, Mingxin Song ${ }^{3}$, Huanhuan Liu' ${ }^{1}$, Bo Wang ${ }^{4}$, Shuchao Wang ${ }^{2}$, Zedong Wang ${ }^{2}$, Hongyu Ma ${ }^{1}$, Zhongyu Li' ${ }^{1}$, Zheng Zeng ${ }^{5}$, Jun Qian ${ }^{2 *}$ and Quan Liü ${ }^{2 *}$

\footnotetext{
${ }^{1}$ College of Life Science, Jilin Agricultural University, Changchun, China, ${ }^{2}$ Key Laboratory of Jilin Province for Zoonosis Prevention and Control, Military Veterinary Institute - Academy of Military Medical Sciences, Changchun, China, ${ }^{3}$ College of Veterinary Medicine, Northeast Agricultural University, Harbin, China, ${ }^{4}$ Department of Pathology, The Second Clinical Medical School of Inner Mongolia University for the Nationalities, Inner Mongolia General Forestry Hospital, Yakeshi, China, ${ }^{5}$ Center for Prevention and Control of Animal Diseases of Chongqing, Chongqing, China
}

Tick-borne diseases are considered as emerging infectious diseases in humans and animals in China. In this study, Ixodes persulcatus $(n=1699)$, Haemaphysalis concinna ( $n=412)$, Haemaphysalis longicornis $(n=390)$, Dermacentor nuttalli $(n=253)$, and Dermacentor silvarum $(n=204)$ ticks were collected by flagging from northeastern China, and detected for infection with Anaplasma, Ehrlichia, Babesia, and Hepatozoon spp. by using nested polymerase chain reaction assays and sequencing analysis. Anaplasma phagocytophilum was detected in all tick species, i.e., I. persulcatus (9.4\%), H. longicornis (1.9\%), H. concinna (6.5\%), D. nuttalli $(1.7 \%)$, and D. silvarum (2.3\%); Anaplasma bovis was detected in $H$. longicornis $(0.3 \%)$ and $H$. concinna (0.2\%); Ehrlichia muris was detected in I. persulcatus $(2.5 \%)$ and $H$. concinna $(0.2 \%)$; Candidatus Neoehrlichia mikurensis was only detected in I. persulcatus (0.4\%). The Ehrlichia variant (GenBank access number KU921424), closely related to Ehrlichia ewingii, was found in $H$. longicornis $(0.8 \%)$ and $H$. concinna $(0.2 \%)$. I. persulcatus was infected with Babesia venatorum (1.2\%), Babesia microti (0.6\%), and Babesia divergens (0.6\%). Additionally, four Babesia sequence variants (GenBank access numbers 862303-862306) were detected in I. persulcatus, H. longicornis, and H. concinna, which belonged to the clusters formed by the parasites of dogs, sheep, and cattle (B. gibsoni, B. motasi, and B. crassa). Two Hepatozoon spp. (GenBank access numbers KX016028 and KX016029) associated with hepatozoonosis in Japanese martens were found in the collected ticks $(0.1-3.1 \%)$. These findings showed the genetic variability of Anaplasma, Ehrlichia, Babesia, and Hepatozoon spp. circulating in ticks in northeastern China, highlighting the necessity for further research of these tick-associated pathogens and their role in human and animal diseases.

Keywords: tick-borne diseases, Anaplasma, Ehrlichia, Babesia, Hepatozoon, northeastern China 


\section{INTRODUCTION}

Ticks are second only to mosquitoes as vectors to transmit viral, bacterial, and protozoan agents in humans and animals, some of which pose a threat to human and animal health and are frequently zoonotic. Among tick-borne bacteria, members of the genera Anaplasma and Ehrlichia of the family Anaplasmataceae cause anaplasmoses and ehrlichioses in humans and animals (Ismail et al., 2010). The two most important species include Ehrlichia chaffeensis, the causative agent of human monocytic ehrlichiosis (HME), and Anaplasma phagocytophilum, the agent of human granulocytic anaplasmosis (HGA). Less commonly, ehrlichiosis induced by Ehrlichia ewingii was first discovered by Buller et al. (1999). Pritt et al. (2011) identified a third species of Ehrlichia muris in patients who had fever, malaise, headache, and lymphopenia in Wisconsin and Minnesota, the United States, and it has also been found in Europe and Asia (Rar et al., 2010; Tateno et al., 2015). Other species that infect animals include A. marginale, Anaplasma centrale, A. ovis, E. canis, and Ehrlichia minasensis (Wen et al., 2003; Kocan et al., 2015; Cabezas-Cruz et al., 2016). Several species have been described in China, such as A. ovis, Anaplasma bovis, and A. platys in Gansu province (Li et al., 2016), A. centrale and E. canis in Fujian province (Ge et al., 2016; Yu et al., 2016), and E. chaffeensis in Guangxi province (Yang et al., 2015b). In Asia, Ixodes persulcatus is considered the primary vector of A. phagocytophilum and E. muris (Jin et al., 2012; Ivanova et al., 2016).

The tick-borne protozoa of the genus Babesia, the causative pathogen of babesiosis in humans and animals, are considered as emerging diseases worldwide. Approximately 100 Babesia species have been identified to infect a broad range of animals, in which malaria-like disorders are induced (Rozej-Bielicka et al., 2015). Babesiosis has a great effect on the animal production and on companion animals; however, human babesiosis has attracted increased attention (Ord and Lobo, 2015). In immunocompetent individuals, the infection is usually asymptomatic, or shows mild, self-resolving symptoms, but babesiosis can be life-threatening in neonates/infants, elderly persons, asplenic patients, and the immunocompromised populations (Fang et al., 2015; Ord and Lobo, 2015). The three most important species to infect humans are $B$. microti, $B$. divergens, and $B$. venatorum. Other species, such as B. ovis, B. major, B. bovis, B. bigemina, B. ovata, $B$. orientalis, $B$. motasi, and $B$. caballi, cause animal infections (Fang et al., 2015). In China, B. microti has been found in rodents in Fujian, Zhejiang, Henan, and Heilongjiang provinces (Sun et al., 2008; Zhao et al., 2013; Chen et al., 2014); B. divergens has been described in I. persulcatus, Haemaphysalis concinna, and $H$. japonica and striped field mice in Heilongjiang province, where $B$. venatorum has also been reported in I. persulcatus (Fang et al., 2015; Jiang et al., 2015). Most ixodid tick species, such as Ixodes scapularis in the United States, Ixodes ricinus in Europe, and I. persulcatus in Asia, can transmit Babesia parasites to their natural hosts (Brasseur and Gorenflot, 1996; Sun et al., 2008; Schulze et al., 2013; Zamoto-Niikura et al., 2016).

Members of Hepatozoon, belonging to Apicomplexa protozoa, parasitize mainly erythrocytes in amphibians, reptiles, and avian hosts, whereas they are found primarily in leukocytes in mammals (Baneth, 2011). In humans, sporadic cases have been reported in Russia (Shuikina et al., 2004). In China, Hepatozoon canis has been reported in dogs from Beijing, Henan, Jiangsu, Shaanxi, and Xingjiang provinces (Xu et al., 2015). Hepatozoon DNA has been detected in I. ricinus, Dermacentor spp., and Haemaphysalis spp., but the vector competence remains to be confirmed (Giannelli et al., 2013; Hamsikova et al., 2016).

The topography of northeastern China includes both plains and mountains, where Changbai Mountains, Da Hinggan Mountains and Xiao Hinggan Mountains are not only the important natural barriers of protecting the ecosystem, but also host to a wide range of natural focal diseases, among which Lyme borreliosis and tick-borne encephalitis are the most common tick-borne diseases (Wu et al., 2013), and emerging tick-borne zoonoses, induced by Rickettsia raoultii, Candidatus Neoehrlichia mikurensis, and B. venatorum, have been reported (Fang et al., 2015). To the best of our knowledge, there is no wide survey of tick-borne pathogens in northeastern China. The objective of this study was to characterize tick-borne bacteria (Anaplasma and Ehrlichia) and protozoa (Babesia and Hepatozoon) in ticks collected from Jilin and Heilongjiang provinces, northeastern China. The data obtained here would contribute to understanding the epidemic and distribution of these tick-borne pathogens, which could be used to design effective control measures for tick-borne diseases in China.

\section{MATERIALS AND METHODS}

\section{Tick Collection and DNA Extraction}

Questing adult ticks were collected by flagging vegetation in Jilin and Heilongjiang provinces, northeastern China, during April-May, 2015 (Liu et al., 2016). The obtained ticks were identified to species using the morphological method (Chen et al., 2010). In total, 253 Dermacentor nuttalli, 204 Dermacentor silvarum, 412 H. concinna, 390 Haemaphysalis longicornis, and $1699 \mathrm{I}$. persulcatus were collected in northeastern China, including 206 D. nuttalli, 175 D. silvarum, $244 \mathrm{H}$. longicornis, and $393 \mathrm{I}$. persulcatus from Jilin Province, and 47 D. nuttalli, 29 D. silvarum, $412 \mathrm{H}$. concinna, $146 \mathrm{H}$. longicornis, and 1276 I. persulcatus from Heilongiiang Province (Liu et al., 2016). The predominant tick species in northeastern China was I. persulcatus (58.0\%), followed by H. concinna (14.0\%), H. longicornis (13.0\%), D. nuttalli (8.0\%), D. silvarum (7.0\%). The detailed information on the collected ticks is given in Supplementary Figure S1.

The ticks were pooled, approximately 15 female ticks per pool, according to their species and sampling site. Total DNA was extracted from crushed ticks using a TIANcombi DNA Lyse \& Det PCR Kit (Tiangen Biotech, Co., Ltd, Beijing, China), and used to detect Anaplasma, Ehrlichia, Babesia, and Hepatozoon DNA by nested PCR assays.

\section{Polymerase Chain Reaction (PCR) Assays}

The involved tick-borne pathogens were detected by nested PCR assays. The used primers were described in previous studies 
(Casati et al., 2006; Kawahara et al., 2006; Tabara et al., 2007; Cicuttin et al., 2014; Aydin et al., 2015; Sumrandee et al., 2015), or designed according to the conserved regions of target genes, as showed in Table $\mathbf{1 .}$

The reactions were conducted in an automatic thermocycler in a total volume of $25 \mu \mathrm{l}$ containing $12.5 \mu \mathrm{l}$ of Premix Taq (TaKaRa Taq Version 2.0 plus dye), $0.5 \mu l$ of each primer ( $5 \mathrm{pmol}), 2.0 \mu \mathrm{l}$ of template DNA ( $\sim 60 \mathrm{ng}$ ), and $9.5 \mu \mathrm{l}$ of distilled water. The reaction conditions of the first-round amplification included $5 \mathrm{~min}$ of pre-denaturation at $94^{\circ} \mathrm{C}$; followed by 30 cycles at $94^{\circ} \mathrm{C}$ for $30 \mathrm{~s}$, annealing for $30 \mathrm{~s}$ at an appropriate temperature, and $72^{\circ} \mathrm{C}$ for $1 \mathrm{~min}$; with a final extension at $72^{\circ} \mathrm{C}$ for $10 \mathrm{~min}$. During the second round of amplification, $1 \mu \mathrm{l}$ of the product from the first-round amplification was used as the template to amplify the target genes using the nested primers; the amplification included 30 cycles of $30 \mathrm{~s}$ at $94^{\circ} \mathrm{C}$, annealing for $30 \mathrm{~s}$ at an appropriate temperature, and $40 \mathrm{~s}$ at $72^{\circ} \mathrm{C}$.

For Anaplasma/Ehrlichia detection, a 345-bp fragment of the $16 \mathrm{~S}$ rRNA was amplified using the universal primers EHR16SD and EHR16SR. The Anaplasma-positive samples could be identified to species by PCR using the species-specific primers, and the Ehrlichia-positive samples were further identified to species by the nested PCR and sequencing.

\section{Phylogenetic Analysis}

The PCR products were purified and sequenced in both directions using the specific primers. Nucleotide sequences were analyzed by BlastN and aligned with ClustalW. The pairwise distance was analyzed using the Kimura's 2-parameter model. Phylogenetic analyses were conducted using the software
MEGA $5^{1}$. The neighbor-joining method was employed to construct a phylogenetic tree. The reliability of branches in the tree was evaluated by bootstraping analysis with 1000 replicates, and the bootstrap value more than $60 \%$ was considered significant.

\section{Statistical Analysis}

The prevalence of infection in ticks was calculated using the program PooledInfRate version 4.0 (Biggerstaff, 2009). Statistical analyses were performed using SPSS version 17.0, SPSS, Inc., Chicago, IL, USA. p-value less than 0.05 was considered statistically significant.

\section{RESULTS}

\section{Anaplasma DNA in Ticks}

Anaplasma DNA was detected in I. persulcatus, $H$. concinna, $H$. longicornis, D. silvarum, and D. nuttalli. Phylogenetic analysis of the partial 16S rRNA gene showed that the detected Anaplasma belonged to A. phagocytophilum and A. bovis (Figure 1). A. phagocytophilum in this study was phylogenetically clustered together with those found in goat from Zhejiang (KP062963), cattle from Hubei in China (KF569911), and deer in Japan (AB196721), which formed a unique haplotype (100\% identity), and was distinct from the other haplotypes, including the isolates in human from USA (U02521), Denmark (AY776165), South Korea (KP306518), and Russia (HM366589; Figure 1; Supplementary Table S1). All the obtained $A$. bovis $16 \mathrm{~S}$ rRNA gene sequences were

${ }^{1}$ http://www.megasoftware.net/

TABLE 1 | Oligonucleotide primers used for the detection of tick-borne pathogens.

\begin{tabular}{|c|c|c|c|c|c|c|}
\hline Pathogen & $\begin{array}{l}\text { Target } \\
\text { gene }\end{array}$ & $\begin{array}{l}\text { Oligonucleotide } \\
\text { primer }\end{array}$ & Primer sequence $\left(5^{\prime} \rightarrow 3^{\prime}\right)$ & $\begin{array}{c}\text { Annealing } \\
\text { temperature } \\
\left({ }^{\circ} \mathrm{C}\right)\end{array}$ & $\begin{array}{l}\text { Amplicon } \\
\text { size (bp) }\end{array}$ & Reference \\
\hline Anaplasma/Ehrlichia spp. & & EHR16SR & TAGCACTCATCGTTTACAGC & & & \\
\hline \multirow[t]{2}{*}{ Anaplasma bovis } & $16 \mathrm{~S}$ rRNA & AB1F & CTCGTAGCTTGCTATGAGAAC & 54 & 551 & Kawahara et al., 2006 \\
\hline & & AB1R & TCTCCCGGACTCCAGTCTG & & & \\
\hline & & ANA-GroR & CGATCAAACTGCATACCATCAGTC & & & \\
\hline \multirow[t]{4}{*}{ Ehrlichia spp. } & groEL & gro607F & GAAGATGCWGTWGGWTGTACKGC & 55 & 730 & Tabara et al., 2007 \\
\hline & & gro1294R & AGMGCTTCWCCTTCWACRTCYTC & & & \\
\hline & & gro677F & ATTACTCAGAGTGCTTCTCARTG & 58 & 364 & \\
\hline & & gro1121R & TGCATACCRTCAGTYTITTCAAC & & & \\
\hline \multirow[t]{4}{*}{ Hepatozoon spp. } & $18 \mathrm{~S}$ rRNA & HepF300 & GCTAATACATGAGCAAAATCTCAA & 54 & 1131 & Sumrandee et al., 2015 \\
\hline & & HepR900 & CGGAA TTAA CCAGACAAAT & & & \\
\hline & & HepF & ATACATGAGCAAAATCTCAAC & 59 & 643 & Aydin et al., 2015 \\
\hline & & HepR & СТTATTATTCCATGCTGCAG & & & \\
\hline
\end{tabular}




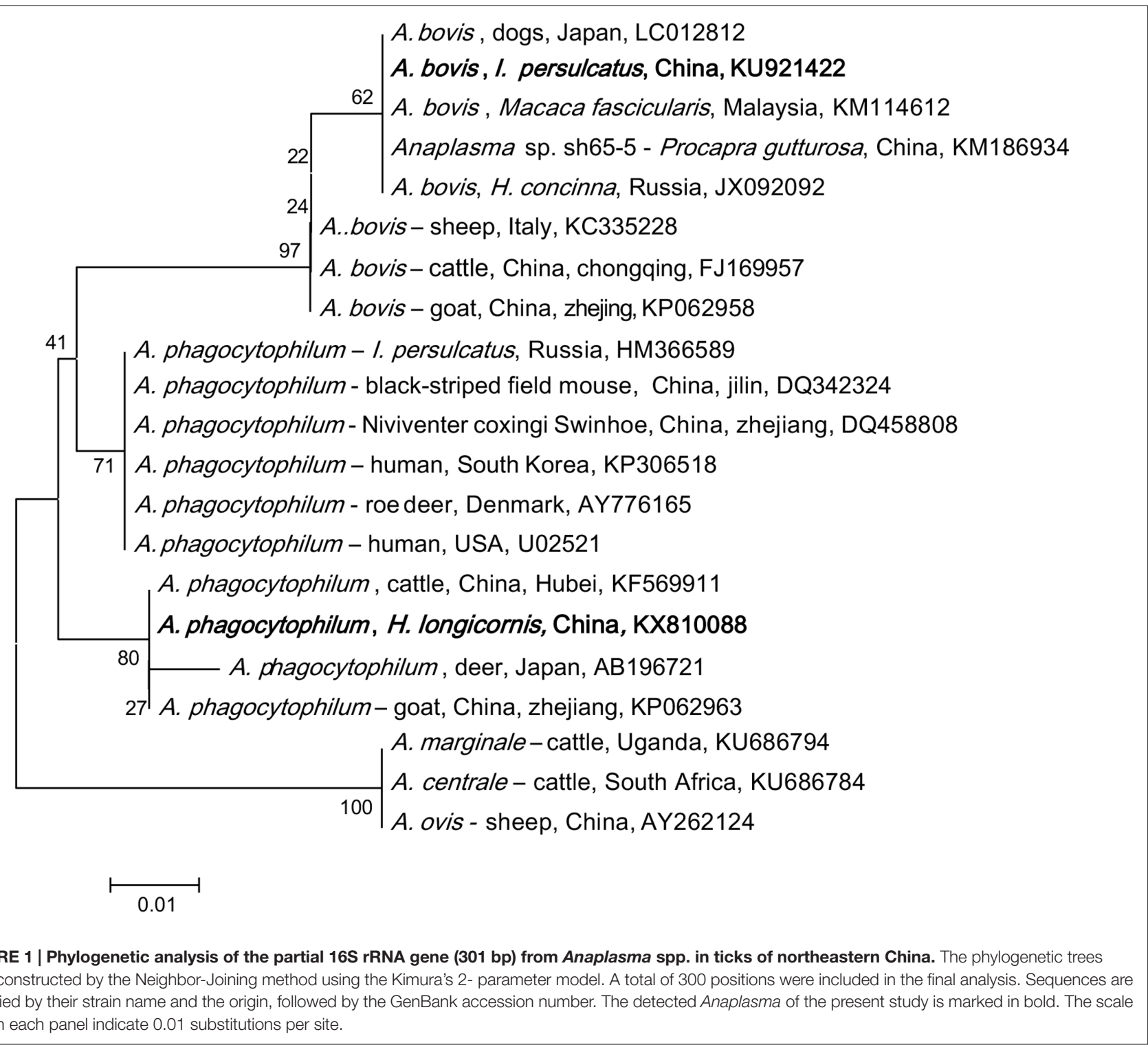

$100 \%$ identical to that found in dogs in Japan (LC012812), Macaca fascicularis in Malaysia (KM114612), and H. concinna in Russia (JX092092), which formed a haplotype different from that detected in cattle in Chongqing (FJ169957), and goat in Zhejiang (KP062958) in China, and sheep in Italy (KC335228; Figure 1).

Anaplasma phagocytophilum was found in all tick species in both provinces, with a higher prevalence in Heilongjiang $(4.5 \%$ in Jilin and $7.2 \%$ in Heilongjiang, $p<0.05$, Supplementary Table S2). High prevalence was detected in I. persulcatus $(9.4 \%$, $p<0.05)$ as comparison with that in D. silvarum (2.3\%), $H$. concinna (1.9\%), and D. nuttalli (1.7\%), suggesting that I. persulcatus may be the primary vector for A. phagocytophilum (Table 2). A. bovis was only found in H. longicornis $(0.7 \%)$ and $H$. concinna $(0.2 \%)$ collected from Heilongjing province, without significant difference between the two species $(p>0.05$, Supplementary Table S1).

\section{Ehrlichia-Specific DNA in Ticks}

Ehrlichia-specific DNA was detected in I. persulcatus, $H$. longicornis, and $H$. concinna, and the phylogenetic analysis of the partial heat shock protein (groEL) gene showed that the detected sequences were grouped with E. muris, Candidatus N. mikurensis, and a possible novel variant (Figure 2).

All the obtained E. muris groEL gene sequences were $100 \%$ identical to those of E. muris detected in I. ricinus from Poland (KF312362), I. persulcatus from Russia (GU358686), and Microtus agrestis from Russia (GU358690, Supplementary Table S3). E. muris was tested in I. persulcatus (1.9\%) and H. concinna $(0.2 \%)$ in Heilongjiang, and I. persulcatus $(4.3 \%)$ in Jilin, with significant difference of infection rate in $I$. persulcatus between the two provinces ( $p<0.05$, Supplementary Table S4). The overall infection rate for E. muris was $2.5 \%$ in I. persulcatus, significantly higher than that in $H$. concinna $(0.2 \%)$ in northeastern China $(p<0.05$, Table 2$)$. 


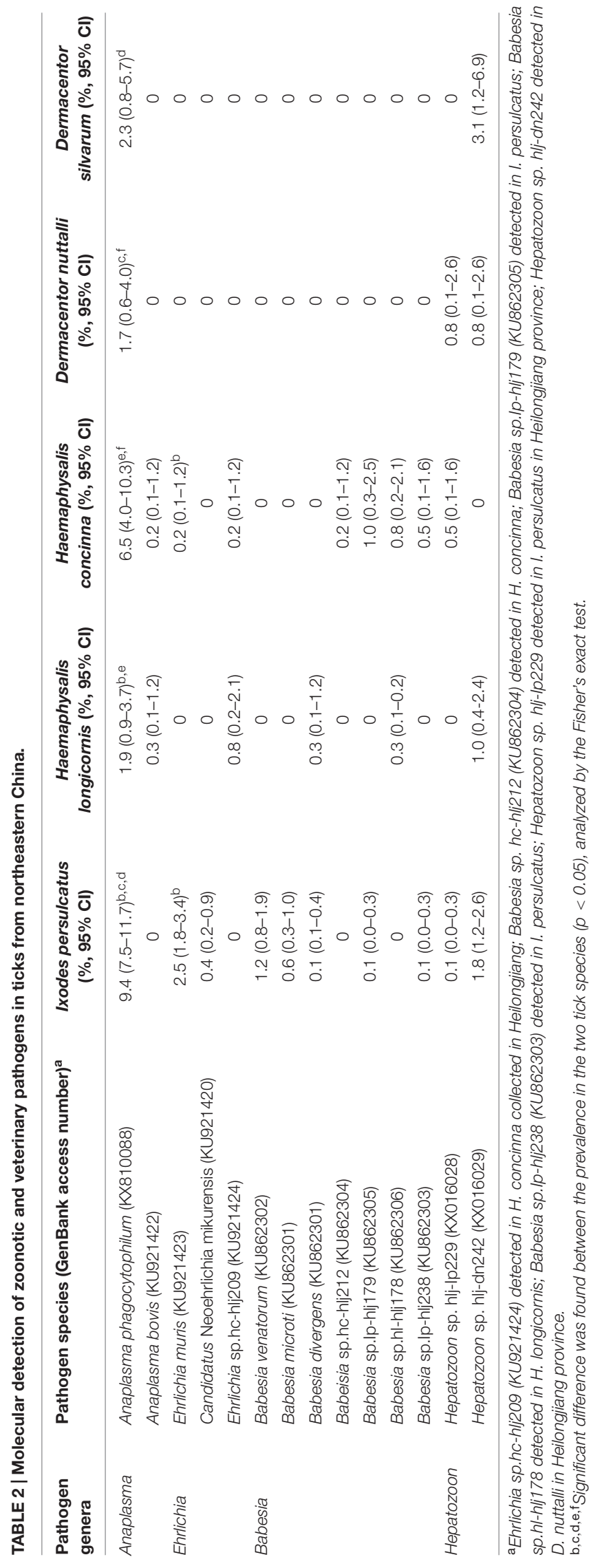

The detected Candidatus N. mikurensis groEL sequences were 100\% identical to the Candidatus N. mikurensis sequences detected in I. persulcatus from Russia (FJ966359) and in wild rodents of Japan (AB204864), and humans from China (JQ359062, Supplementary Table S3), which were phylogenetically clustered, distinctive from the European countries, including Hungary, Germany, Netherlands, and Italy (Figure 2). Candidatus N. mikurensis were only found in I. persulcatus in Jilin (0.3\%) and Heilongjiang (0.5\%), and no significant difference was found between the two provinces ( $p>0.05$, Supplementary Table S4).

The typical variant, Ehrlichia sp. Kh-Hj27 found in Russia, which showed the highest similarity (96\%) to that of E. ewingii (AF195273, Supplementary Table S3), was also detected in both H. longicornis $(2.2 \%)$ and H. concinna $(0.2 \%)$ in Heilongjiang province, clustered together with one more Ehrlichia genetic variant (Am-Hc79, JX092091) detected in H. concinna in Russia (97\% identity), thus forming a separate branch on the phylogenetic tree (Supplementary Table S3; Figure 2).

\section{Babesia DNA in Ticks}

Babesia DNA was detected in I. persulcatus, H. longicornis, and $H$. concinna in Jilin and Heilongjiang provinces of northeastern China, but positive results was obtained neither from $D$. nuttalli nor from $D$. silvarum. Phylogenetic analysis showed that the Babesia species in ticks from Heilongjiang and Jilin provinces were clustered together with $B$. venatorum, B. microti, $B$. divergens, and the four Babesia genetic variants belonged to the carnivores, cattle and small ruminants groups (Figure 3), with the DNA sequence homology of $99-100 \%$ (GenBank accession numbers: KU862300-KU862306).

The obtained gene sequences of $B$. venatorum $18 \mathrm{~S}$ rRNA were $100 \%$ identical to each other and to those found in I. ricinus of France (FJ215873), and in I. persulcatus of Russia (KJ486557) and Mongolia (LC005775, Supplementary Table S5), and the phylogenetic analysis showed that the $B$. venatorum from Jilin and Heilongjiang provinces of China and Europe clustered in the same clade, but distinct from other Babesia species (Figure 3). Only $I$. persulcatus $(1.2 \%)$ was detected to be infected with $B$. venatorum, with a prevalence of $0.3 \%$ in Jilin and $1.6 \%$ in Heilongjiang ( $p<0.05$, Table 2; Supplementary Table S6). Other ticks, including Dermacentor and Haemaphysalis, were detected negative.

Nine I. persulcatus pools were detected positive for B. microti, whose sequences completely matched the B. microti strains isolated from humans in the United States (AF231348), tundra vole of Russia (AY943957), and from I. persulcatus of Mongolia (LC005772, Supplementary Table S5), the phylogenetic analysis indicated that these isolates were clustered in the same clade (Figure 3). The overall prevalence of $B$. microti was $0.6 \%$ in I. persulcatus, with a prevalence of $1.1 \%$ in Jilin and $0.4 \%$ in Heilongjiang ( $p>0.05$, Supplementary Table S6). The other tick species were tested negative. Despite the high similarity of B. microti found in northeastern China to highly pathogenic strains, human babesiosis caused by B. microti has not been confirmed in northern China to date. We cannot, however, rule out the existence of human cases. 


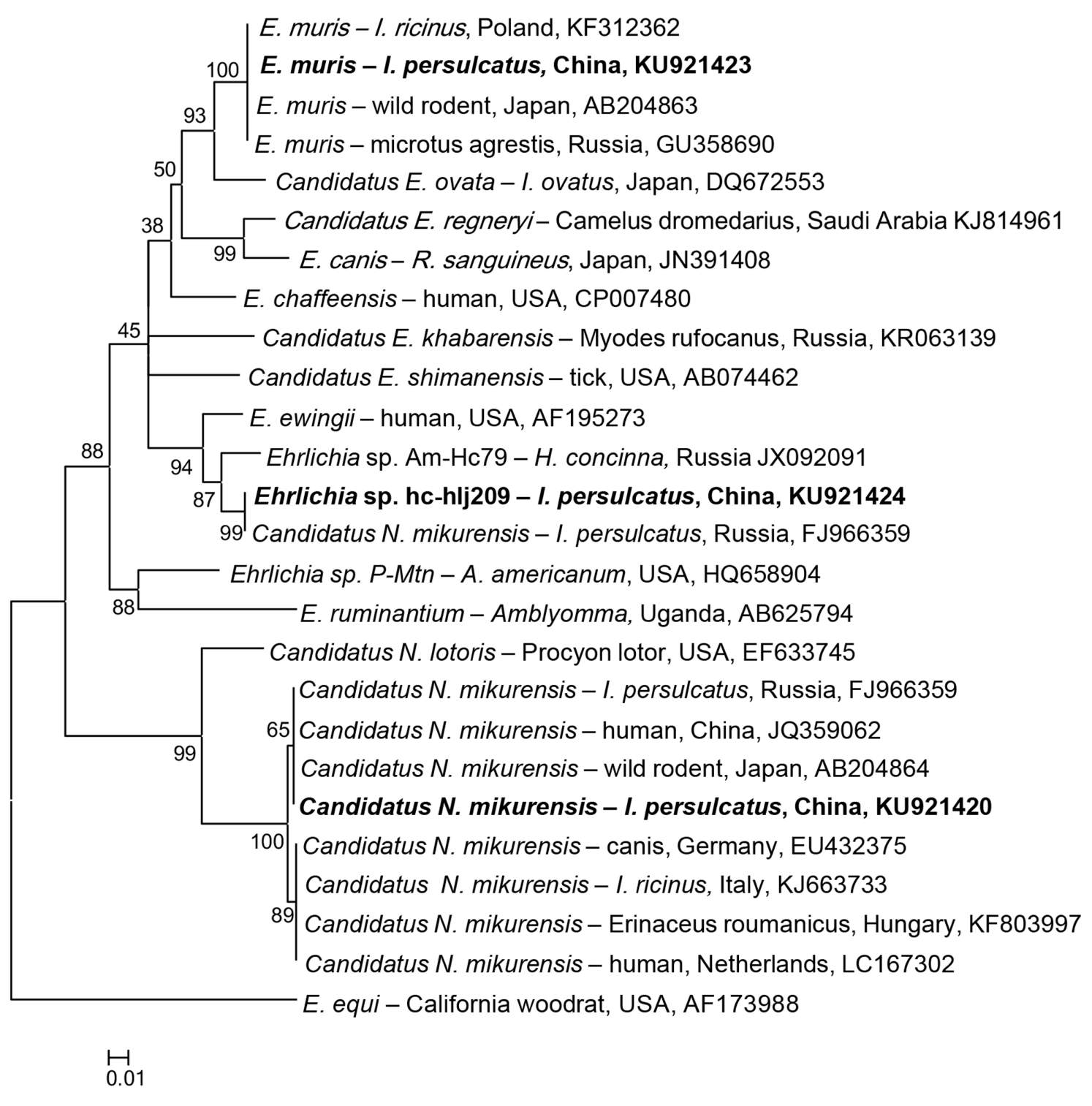

FIGURE 2 | Phylogenetic analysis of the partial groEL gene (287 bp) from Ehrlichia spp. in ticks from northeastern China. The phylogenetic trees were constructed by the Neighbor-Joining method using the Kimura's 2- parameter model. A total of 284 positions were included in the final analysis. Sequences are identified by their strain name and the origin, followed by the GenBank accession number. The detected Ehrlichia of the present study is marked in bold. The scale bars in each panel indicate 0.01 substitutions per site.

Two H. longicornis pools and one I. persulcatus pool in Heilongjiang province were tested positive for $B$. divergens variant, showing a prevalence of 0.7 and $0.3 \%$, respectively, (Supplementary Table S6). The obtained 18S rRNA gene sequences were $100 \%$ identical to the strains isolated from I. persulcatus in Russia (GU057385 and KJ486559, Supplementary Table S5), but differed from both the European $B$. divergens and B. capreoli isolates, and the Babesia sp. MO1 isolate recovered from humans in the United States, and formed a separate clade (Figure 3). Thus, we cannot group this genetic variant with any particular species.

In addition, four Babesia sequence variants were detected in H. longicornis, H. concinna, and I. persulcatus (Table 2), which were closely related to the groups of carnivores, cattle and small ruminant Babesia (Figure 3). These variants have also been found in Russia (GU057382, KJ486566, KJ486568, and KJ486569), with the 18S rRNA gene sequences of $100 \%$ identify (Supplementary Table S5), but their mammalian host species remain unknown (Rar et al., 2011).

\section{Hepatozoon DNA in Ticks}

The Hepatozoon DNA was detected in D. nuttalli, D. silvarum, $H$. concinna, $H$. longicornis, and $I$. persulcatus, which were phylogenetically divided into two groups (Figure 4), and were identical (99-100\%) to Hepatozoon isolates JM-6 (FJ595132), 


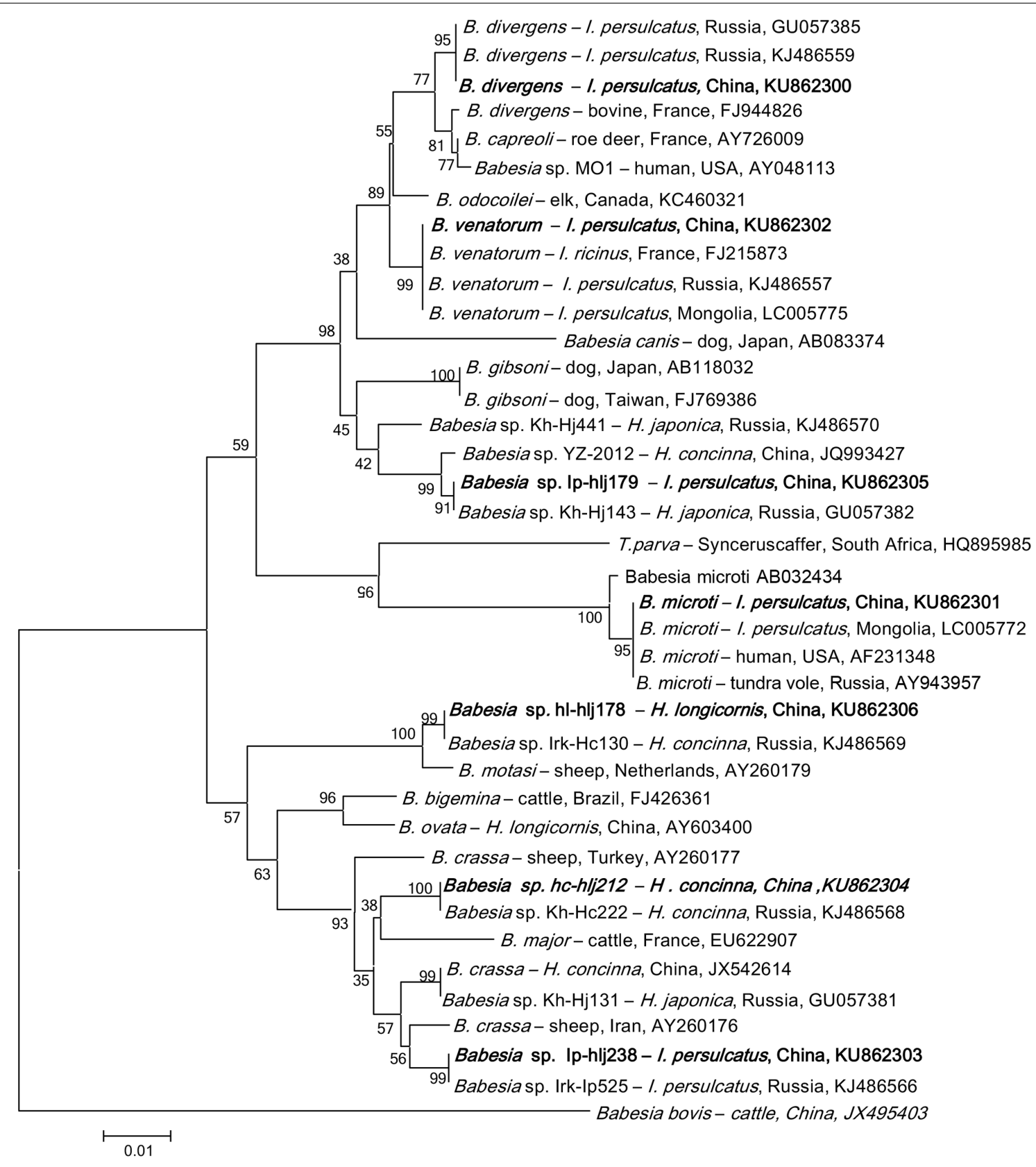

FIGURE 3 | Phylogenetic analysis of the partial 18S rRNA gene (740 bp) from Babesia spp. in ticks from northeastern China. The phylogenetic trees were constructed by the Neighbor-Joining method using the Kimura's 2- parameter model. A total of 721 positions were included in the final analysis. Sequences are identified by their strain name and the origin, followed by the GenBank accession number. The detected Babesia of the present study is marked in bold. The scale bars in each panel indicate 0.01 substitutions per site.

and JM-7 (FJ595133) (Supplementary Table S7), isolated from Japanese martens (Martes melampus melampus) (Kubo et al., 2009). The prevalences of Hepatozoon sp. were very low in all tick species, ranging from 0.1 to $4.4 \%$, and no significant difference was found $(p>0.05$, Table 2 , Supplementary Table S8). No other Hepatozoon species was detected in ticks from Jilin and Heilongjiang provinces of Northeastern China.

\section{DISCUSSION}

Several species of Anaplasma, including A. phagocytophilum, A. bovis, A. marginale, and A. ovis, have been described in China (Li et al., 2016; Zhang et al., 2016). A. phagocytophilum is considered as an emerging human pathogen of public health importance, which is naturally maintained in tick-mammal cycles, and has been found in sheep, goats, cattle, rabbits, 


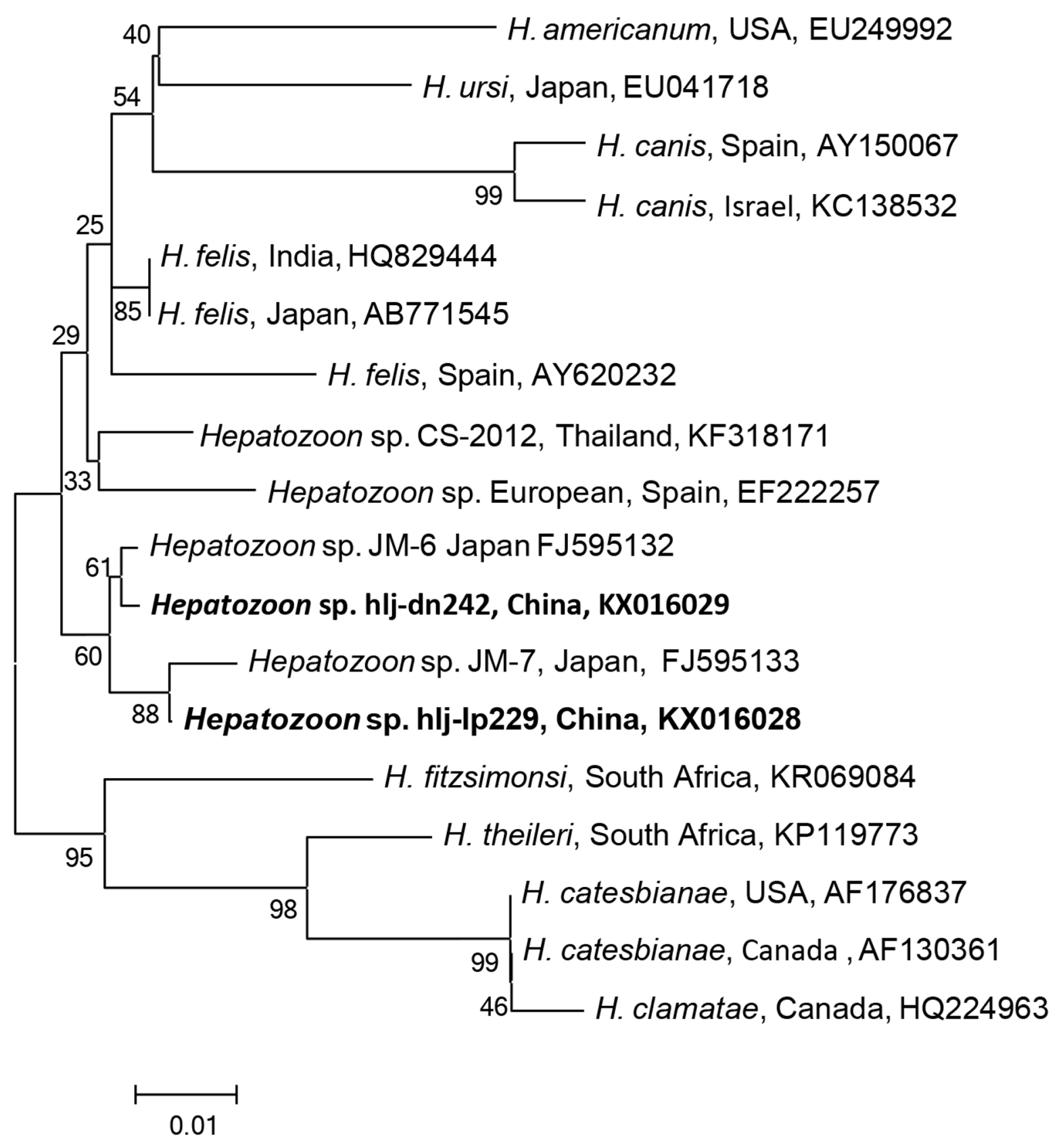

FIGURE 4 | Phylogenetic analysis of the partial 18S rRNA gene (343 bp) from Hepatozoon spp. in ticks from northeastern China. The phylogenetic trees were constructed by the Neighbor-Joining method using the Kimura's 2- parameter model. A total of 341 positions were included in the final analysis. Sequences are identified by their strain name and the origin, followed by the GenBank accession number. The detected Hepatozoon of the present study is marked in bold. The scale bars in each panel indicate 0.01 substitutions per site.

and rodents (Jin et al., 2012). Although, A. phagocytophilum was detected in the genera of Ixodes, Dermacentor, and Haemaphysalis, I. persulcatus may play the most important role in the transmission of the bacterium in northeastern China, due to the high infection rate in the tick species and its high abundance. In Hebei province, A. phagocytophilum was detected in $H$. longicornis and $D$. nuttalli, where $H$. longicornis is the predominant tick species for the transmission of this pathogen
(Yaxue et al., 2011). Phylogenetically, A. phagocytophilum detected in the study was more likely to infect ruminants (Yang et al., 2015a).

Anaplasma bovis has a wide host range, whose susceptible species include cattle, goats, dogs, cats, and deer (Kocan et al., 2015). In recent years, molecular detection of $A$. bovis infection showed an overall prevalence of $10-16 \%$ in goats, and $9.7 \%$ in sheep in China (Liu et al., 2012; Zhang et al., unpublished). Both 
$H$. concinna and $H$. longicornis ticks were found to be infected with $A$. bovis, suggesting these two tick species may be responsible for the transmission of $A$. bovis between ticks and mammals, but this assumption needs further experimental evidence.

Ehrlichia spp. are obligate intracellular bacteria residing within the cytoplasmic vacuoles of monocytes, granulocytes, or platelets of humans and animals, and can cause illnesses with fever, leukopenia, and thrombocytopenia (Dumler and Bakken, 1995). Serological and molecular evidences show a wide distribution of the bacteria infection in ticks, animals, and humans (Yu et al., 2016). In China, E. chaffeensis was detected in Amblyomma testudinarium, $H$. yeni, and I. persulcatus (Cao et al., 2000; Zhang et al., 2014). E. canis has been identified in Rhipicephalus sanguineus and Rhipicephalus microplus (Wen et al., 2003), and human infection usually presents fever, malaise, thrombocytopenia, and lymphopenia (Johnson et al., 2015). E. muris has been detected in $R$. microplus in Hunan (Yu et al., 2016). In the present study, we first detected E. muris in I. persulcatus collected from Jilin and Heilongjiang provinces of northeastern China, with a prevalence of 1.9-4.3\%, showing that I. persulcatus may be a vector for this bacterium in northeastern China. Further studies are needed to assess the possible emergence of the infections in northeastern China.

Candidatus N. mikurensis is an emerging tick-borne pathogen causing neoehrlichiosis, whose clinical symptoms may include fever, localized pain in muscles and/or joints, vascular and thromboembolic events (Silaghi et al., 2016). In China, the human infection was first reported in Heilongjiang province of northeastern China in Li et al. (2012), and the bacterium may have wide geographic distribution in China (Li et al., 2013). Only I. persulcatus was positive for Candidatus N. mikurensis in this study, implying that $I$. persulcatus may be a vector for this bacterium in northeastern China.

Babesiosis, the causative pathogens including B. microti, $B$. venatorum, and $B$. divergens in humans, is considered an emerging threat in China, where there are approximately 1.3 billion people at risk of infection (Qi et al., 2011; Jiang et al., 2015; Vannier and Krause, 2015). B. microti was detected in I. persulcatus, H. longicornis, and H. concinna in Fujian, Zhejiang, Henan, and Heilongjiang provinces (Saito-Ito et al., 2008; Sun et al., 2008; Zhou et al., 2014). B. venatorum has been reported in I. persulcatus ticks collected from forested areas of northeastern China (Jiang et al., 2015). Other species of Babesia, including B. ovis, B. major, B. ovata, B. orientalis, B. motasi, and B. caballi, have not been shown to infect humans (Fang et al., 2015). In the present study, we found $B$. divergens, $B$. microti, and $B$. venatorum in $I$. persulcatus, and also first detected $B$. divergens in $H$. longicornis, indicating that $I$. persulcatus may be the main vector for the Babesia species of human babesiosis in northeastern China, since only two $H$. longicornis pools were positive for this parasite.

Previous studies have shown that B. microti is the predominant species in southeastern and northeastern of China while $B$. divergens may be the main pathogen in Inner Mongolia, and Xinjiang Uygur Autonomous Region of China (Zhou et al., 2014). In this study, of the 47 Babesia-positive samples, 19 (40.2\%) were B. venatorum, followed by 9 (19.1\%)
B. microti, $3(6.4 \%)$ B. divergens, and 16 (34.3\%) sequence variants, suggesting that $B$. venatorum may be the predominant species responsible for human babesiosis in northeastern China.

Additionally, several sequence variants, which are closely related to the groups of carnivores, cattle and small ruminant Babesia, have also been found in the present study. For example, Babesia sp. Ip-hlj179 was phylogenetically associated with B. gibsoni; Babesia sp. Ip-hlj179 was related to B. motasi; Babesia sp. Ip-hlj238 was related to B. crassa; and Babesia sp. Iphlj212 was related to $B$. major (Figure 3 ). Thus, it is necessary to monitor these variants infection in domestic animals in northeastern China. Isolation of parasites and identification of transmission vector should also be included. Interestingly, a high genetic variability of Babesia has been described in Russia, which included all variants found in northeastern China (Rar et al., 2014), and four variants were detected in Heilongjiang province while only two variants were found in Jilin province. These data showed the cross-border spread of Babesia in northeastern China may occur.

More than 300 Hepatozoon species have been identified in amphibians, reptiles, birds, marsupials, and mammals (Smith, 1996). Of these, more than 120 species infect snakes, and approximately 50 have been described in mammals. Ticks and other blood-sucking arthropods may serves as definitive hosts for Hepatozoon spp. Unlike, other vector-borne pathogens that are transmitted via the bite of arthropods, the vertebrate host becomes infected by ingestion of the arthropods that contains mature oocysts. Three species $H$. canis, $H$. americanum, and $H$. felis can cause hepatozoonosis in dogs and cats, showing different clinical symptoms. $H$. canis is primarily found in hemolymphatic tissues, causing fever, lethargy, weight loss, anemia, and hyperglobulinemia in dogs, while $H$. americanum infects mainly muscular tissues, causing myositis and lameness (Baneth, 2011). There is only one report of infection with a Hepatozoon sp. in a person from Russia; the patient was anemic and icteric, and gamonts were detected in the blood (Shuikina et al., 2004).

In China, $H$. canis infection in dogs was detected $1.1 \%$ in Jiangsu, $1.2 \%$ in Xinjiang, $8.9 \%$ in Shaanxi, and $4.9 \%$ in Henan and Beijing (Xu et al., 2015). A new species, H. chinensis has been found in king rat snakes (Elaphe carinata) from Shanghai (Han et al., 2015). In this study, two Hepatozoon species, the most closely related to the isolates of Japanese martens, were found in ticks in northeastern China, and both of them were detected in Ixodes, Haemaphysalis, and Dermacentor ticks, suggesting that specificity for the final host may be low in Hepatozoon; however, the intermediate hosts and the resulting disease still remain to be determined.

Not only the high infection rates but also the high abundance of $I$. persulcatus makes it the most important vector tick in the area. Moreover, Candidatus N. mikurensis, B. venatorum, and B. microti were only detected in $I$. persulcatus. These findings show that $I$. persulcatus may be an important vector of tick-borne bacteria and protozoa in northeastern China.

In summary, we detected four species of bacteria and three species of protozoa in four tick species in northeastern China, including A. phagocytophilum, A. bovis, E. muris, Candidatus 
N. mikurensis, B. venatorum, B. microti, and B. divergens, which are associated with emerging diseases in humans and/or animals. Additionally, four Babesia sequence variants, and two Hepatozoon sp. were also found. These findings showed the genetic variability of Anaplasma, Ehrlichia, Babesia, and Hepatozoon spp. circulating in ticks in northeastern China, highlighting the need for further research of these tick-associated pathogens and their role in human and animal diseases. Further studies will be necessary to confirm the vectorial capacity of ticks, to improve understanding of the epidemiology of these tickborne diseases, and to monitor emerging tick-borne pathogens and factors influencing their prevalence, which will facilitate implementing integrated strategies for controlling ticks and tickborne pathogens in China.

\section{AUTHOR CONTRIBUTIONS}

JQ and QL designed the study in collaboration with FW, MS, and HL. HM conducted the fieldwork with assistance from MS, ZZ, and QL. HL, BW, ZW, and ZL conducted the laboratory work; HL and SW conducted the statistical analysis and drafted the manuscript. FW and MS contributed to the interpretation of

\section{REFERENCES}

Aydin, M. F., Sevinc, F., and Sevinc, M. (2015). Molecular detection and characterization of Hepatozoon spp. in dogs from the Central part of Turkey. Ticks Tick Borne Dis. 6, 388-392. doi: 10.1016/j.ttbdis.2015.03.004

Baneth, G. (2011). Perspectives on canine and feline hepatozoonosis. Vet. Parasitol. 181, 3-11. doi: 10.1016/j.vetpar.2011.04.015

Biggerstaff, B. J. P. (2009). Version 4.0: A Microsoft Office Excel Add-In to Compute Prevalence Estimates from Pooled Samples. Fort Collins, CO: Centers for Disease Control and Prevention.

Brasseur, P., and Gorenflot, A. (1996). Human babesial infections in Europe. Rocz. Akad. Med. Bialymst. 41, 117-122.

Buller, R. S., Arens, M., Hmiel, S. P., Paddock, C. D., Sumner, J. W., Rikhisa, Y., et al. (1999). Ehrlichia ewingii, a newly recognized agent of human ehrlichiosis. N. Engl. J. Med. 341, 148-155. doi: 10.1056/NEJM199907153410303

Cabezas-Cruz, A., Zweygarth, E., Vancova, M., Broniszewska, M., Grubhoffer, L., Passos, L. M., et al. (2016). Ehrlichia minasensis sp. nov., a new species within the genus Ehrlichia isolated from the tick Rhipicephalus microplus. Int. J. Syst. Evol. Microbiol. doi: 10.1099/ijsem.0.000895 [Epub ahead of print].

Cao, W. C., Gao, Y. M., Zhang, P. H., Zhang, X. T., Dai, Q. H., Dumler, J. S., et al. (2000). Identification of Ehrlichia chaffeensis by nested PCR in ticks from Southern China. J. Clin. Microbiol. 38, 2778-2780.

Casati, S., Sager, H., Gern, L., and Piffaretti, J. C. (2006). Presence of potentially pathogenic Babesia sp. for human in Ixodes ricinus in Switzerland. Ann. Agric. Environ. Med. 13, 65-70.

Chen, Z., Liu, Q., Liu, J. Q., Xu, B. L., Lv, S., Xia, S., et al. (2014). Tick-borne pathogens and associated co-infections in ticks collected from domestic animals in central China. Parasit. Vectors. 7:237. doi: 10.1186/1756-3305-7-237

Chen, Z., Yang, X., Bu, F., Yang, X., and Liu, J. (2010). Ticks (acari: ixodoidea: argasidae, ixodidae) of China. Exp. Appl. Acarol. 51, 393-404. doi: 10.1007/ s10493-010-9335-2

Cicuttin, G. L., Brambati, D. F., Eugui, J. I. R., Lebrero, C. G., Salvo, M. N. D., Beltrán, F. J., et al. (2014). Molecular characterization of Rickettsia massiliae and Anaplasma platys infecting Rhipicephalus sanguineus ticks and domestic dogs, Buenos Aires (Argentina). Ticks Tick Borne Dis. 5, 484-488. doi: 10.1016/ j.ttbdis.2014.03.001

Dumler, J. S., and Bakken, J. S. (1995). Ehrlichial diseases of humans: emerging tick-borne infections. Clin. Infect. Dis. 20, 1102-1110. doi: 10.1093/clinids/20.5. 1102 the data. All authors contributed to the manuscript editing and approved the final manuscript.

\section{FUNDING}

This study was supported by grant from the National Science \& Technology Pillar Program during the Twelfth Five-year Plan Period (2013BAD12B04), the Military Medical Health project in China (13CXZ024), the National Key Research Program during the Thirteenth Five-year Plan Period (2016YFC1201602) and the Science and Technology Basic Work Program from the Ministry of Science and Technology of China (2013FY113600).

\section{SUPPLEMENTARY MATERIAL}

The Supplementary Material for this article can be found online at: http://journal.frontiersin.org/article/10.3389/fmicb. 2016.01913/full\#supplementary-material

FIGURE S1 | Sampling sites of ticks collected in Jilin and Heilongjiang provinces of northeastern China. Tick species and the number are shown.

Fang, L. Q., Liu, K., Li, X. L., Liang, S., Yang, Y., Yao, H. W., et al. (2015). Emerging tick-borne infections in mainland China: an increasing public health threat. Lancet Infect. Dis. 15, 1467-1479. doi: 10.1016/S1473-3099(15)00177-2

Ge, Y., Yin, H., Rikihisa, Y., Pan, W., and Yin, H. (2016). Molecular detection of tick-borne rickettsiales in goats and sheep from Southeastern China. Vector Borne Zoonotic Dis. 16, 309-316. doi: 10.1089/vbz.2015.1884

Giannelli, A., Ramos, R. A., Dantas-Torres, F., Mencke, N., Baneth, G., and Otranto, D. (2013). Experimental evidence against transmission of Hepatozoon canis by Ixodes ricinus. Ticks Tick Borne Dis. 4, 391-394. doi: 10.1016/j.ttbdis. 2013.03.001

Hamsikova, Z., Silaghi, C., Rudolf, I., Venclikova, K., Mahrikova, L., Slovak, M., et al. (2016). Molecular detection and phylogenetic analysis of Hepatozoon spp. in questing Ixodes ricinus ticks and rodents from Slovakia and Czech Republic. Parasitol. Res. 115, 3897-3904. doi: 10.1007/s00436-016-5156-5

Han, H., Wu, Y., Dong, H., Zhu, S., Li, L., Zhao, Q., et al. (2015). First report of Hepatozoon (Apicomplexa: Adeleorina) from king ratsnakes (Elaphe carinata) in Shanghai, with description of a new species. Acta Parasitol. 60, 266-274. doi: 10.1515/ap-2015-0038

Ismail, N., Bloch, K. C., and McBride, J. W. (2010). Human ehrlichiosis and anaplasmosis. Clin. Lab. Med. 30, 261-292. doi: 10.1016/j.cll.2009.10.004

Ivanova, A., Geller, J., Katargina, O., Värv, K., Lundkvist, A., and Golovljova, I. (2016). Detection of Candidatus Neoehrlichia mikurensis and Ehrlichia muris in Estonian ticks. Ticks Tick Borne Dis. doi: 10.1016/j.ttbdis.2016.08.010 [Epub ahead of print].

Jiang, J. F., Zheng, Y. C., Jiang, R. R., Li, H., Huo, Q. B., Jiang, B. G., et al. (2015). Epidemiological, clinical, and laboratory characteristics of 48 cases of "Babesia venatorum" infection in China: a descriptive study. Lancet Infect. Dis. 15, 196-203. doi: 10.1016/S1473-3099(14)71046-1

Jin, H., Wei, F., Liu, Q., and Qian, J. (2012). Epidemiology and control of human granulocytic anaplasmosis: a systematic review. Vector Borne Zoonotic Dis. 12, 269-274. doi: 10.1089/vbz.2011.0753

Johnson, D. K., Schiffman, E. K., Davis, J. P., Neitzel, D. F., Sloan, L. M., Nicholson, W. L., et al. (2015). Human infection with Ehrlichia muris-like pathogen, United States, 2007-2013. Emerg. Infect. Dis. 21, 1794-1799. doi: 10.3201/eid2110. 150143

Kawahara, M., Rikihisa, Y., Lin, Q., Isogai, E., Tahara, K., Itagaki, A., et al. (2006). Novel genetic variants of Anaplasma phagocytophilum, Anaplasma bovis, Anaplasma centrale, and a novel Ehrlichia sp. in wild deer and ticks on two major islands in Japan. Appl. Environ. Microbiol. 72, 1102-1109. 
Kocan, K. M., de la Fuente, J., and Cabezas-Cruz, A. (2015). The genus Anaplasma: new challenges after reclassification. Rev. Sci. Tech. 34, 577-586.

Kubo, M., Nagataki, M., Agatsuma, T., Sakai, H., Masegi, T., Panciera, R. J., et al. (2009). Parasitological and molecular features of the Hepatozoon species in the myocardium of Japanese Martens (Martes melampus melampus). J. Parasitol. 95, 1496-1502. doi: 10.1645/GE-2187.1

Li, H., Jiang, J., Tang, F., Sun, Y., Li, Z., Zhang, W., et al. (2013). Wide distribution and genetic diversity of "Candidatus Neoehrlichia mikurensis" in rodents from China. Appl. Environ. Microbiol. 79, 1024-1027. doi: 10.1128/AEM.02917-12

Li, H., Jiang, J. F., Liu, W., Zheng, Y. C., Huo, Q. B., Tang, K., et al. (2012). Human infection with Candidatus Neoehrlichia mikurensis, China. Emerg. Infect. Dis. 18, 1636-1639. doi: 10.3201/eid1810.120594

Li, Y., Chen, Z., Liu, Z., Liu, J., Yang, J., Li, Q., et al. (2016). Molecular survey of Anaplasma and Ehrlichia of red deer and sika deer in Gansu, China in 2013. Transbound. Emerg. Dis. 63, e228-e236. doi: 10.1111/tbed.12335

Liu, H., Li, Q., Zhang, X., Li, Z., Wang, Z., Song, M., et al. (2016). Characterization of rickettsiae in ticks in northeastern China. Parasit. Vectors 9:498. doi: 10.1186/ s13071-016-1764-2

Liu, Z., Ma, M., Wang, Z., Wang, J., Peng, Y., Li, Y., et al. (2012). Molecular survey and genetic identification of Anaplasma species in goats from central and southern China. Appl. Environ. Microbiol. 78, 464-470. doi: 10.1128/AEM. 06848-11

Ord, R. L., and Lobo, C. A. (2015). Human babesiosis: pathogens, prevalence, diagnosis and treatment. Curr. Clin. Microbiol. Rep. 2, 173-181. doi: 10.1007/ s40588-015-0025-Z

Pritt, B. S., Sloan, L. M., Johnson, D. K., Munderloh, U. G., Paskewitz, S. M., McElroy, K. M., et al. (2011). Emergence of a new pathogenic Ehrlichia species, Wisconsin and Minnesota, 2009. N. Engl. J. Med. 365, 422-429. doi: 10.1056/ NEJMoa1010493

Qi, C., Zhou, D., Liu, J., Cheng, Z., Zhang, L., Wang, L., et al. (2011). Detection of Babesia divergens using molecular methods in anemic patients in Shandong Province, China. Parasitol. Res. 109, 241-245. doi: 10.1007/s00436-011-2382-8

Rar, V. A., Epikhina, T. I., Livanova, N. N., and Panov, V. V. (2011). Genetic diversity of Babesia in Ixodes persulcatus and small mammals from North Ural and West Siberia, Russia. Parasitology 138, 175-182. doi: 10.1017/ S0031182010001162

Rar, V. A., Epikhina, T. I., Suntsova, O. V., Kozlova, I. V., Lisak, O. V., Pukhovskaya, N. M., et al. (2014). Genetic variability of Babesia parasites in Haemaphysalis spp. and Ixodes persulcatus ticks in the Baikal region and Far East of Russia. Infect. Genet. Evol. 28, 270-275. doi: 10.1016/j.meegid.2014.10. 010

Rar, V. A., Livanova, N. N., Panov, V. V., Doroschenko, E. K., Pukhovskaya, N. M., Vysochina, N. P., et al. (2010). Genetic diversity of Anaplasma and Ehrlichia in the Asian part of Russia. Ticks Tick Borne Dis. 1, 57-65. doi: 10.1016/j.ttbdis. 2010.01.002

Rozej-Bielicka, W., Stypulkowska-Misiurewicz, H., and Golab, E. (2015). Human babesiosis. Przegl. Epidemiol. 69, 489-494.

Saito-Ito, A., Takada, N., Ishiguro, F., Fujita, H., Yano, Y., Ma, X. H., et al. (2008). Detection of Kobe-type Babesia microti associated with Japanese human babesiosis in field rodents in central Taiwan and southeastern mainland China. Parasitology 135, 691-699. doi: 10.1017/S0031182008004356

Schulze, T. L., Jordan, R. A., Healy, S. P., and Roegner, V. E. (2013). Detection of Babesia microti and Borrelia burgdorferi in host-seeking Ixodes scapularis (Acari: Ixodidae) in Monmouth County, New Jersey. J. Med. Entomol. 50, 379-383. doi: 10.1603/ME12088

Shuikina, E. E., Beier, T. V., Sergiev, V. P., and Iastrebova, R. I. (2004). Detection of hemogregarin of the genus Hepatozoon in patients in Russia. Med. Parasitol. 4, 3-6.

Silaghi, C., Beck, R., Oteo, J. A., Pfeffer, M., and Sprong, H. (2016). Neoehrlichiosis: an emerging tick-borne zoonosis caused by Candidatus Neoehrlichia mikurensis. Exp. Appl. Acarol. 68, 279-297. doi: 10.1007/s10493015-9935-y

Smith, T. G. (1996). The genus Hepatozoon (Apicomplexa: Adeleina). J. Parasitol. 82, 565-585. doi: $10.2307 / 3283781$

Sumrandee, C., Baimai, V., Trinachartvanit, W., and Ahantarig, A. (2015). Hepatozoon and Theileria species detected in ticks collected from mammals and snakes in Thailand. Ticks Tick Borne Dis. 6, 309-315. doi: 10.1016/j.ttbdis.2015. 02.003
Sun, Y., Liu, G., Yang, L., Xu, R., and Cao, W. (2008). Babesia microti-like rodent parasites isolated from Ixodes persulcatus (Acari: Ixodidae) in Heilongjiang Province, China. Vet. Parasitol. 156, 333-339. doi: 10.1016/j.vetpar.2008. 05.026

Tabara, K., Arai, S., Kawabuchi, T., Itagaki, A., Ishihara, C., Satoh, H., et al. (2007). Molecular survey of Babesia microti, Ehrlichia species and Candidatus Neoehrlichia mikurensis in wild rodents from Shimane Prefecture, Japan. Microbiol. Immunol. 51, 359-367.

Tateno, M., Sunahara, A., Nakanishi, N., Izawa, M., Matsuo, T., Setoguchi, A., et al. (2015). Molecular survey of arthropod-borne pathogens in ticks obtained from Japanese wildcats. Ticks Tick Borne Dis. 6, 281-289. doi: 10.1016/j.ttbdis.2015. 01.009

Vannier, E., and Krause, P. J. (2015). Babesiosis in China, an emerging threat. Lancet Infect. Dis. 15, 137-139. doi: 10.1016/S1473-3099(14)71062-X

Wen, B., Cao, W., and Pan, H. (2003). Ehrlichiae and ehrlichial diseases in china. Ann. N. Y. Acad. Sci. 990, 45-53. doi: 10.1111/j.1749-6632.2003. tb07335.x

Wu, X. B., Na, R. H., Wei, S. S., Zhu, J. S., and Peng, H. J. (2013). Distribution of tick-borne diseases in China. Parasit. Vectors 6:119. doi: 10.1186/1756-3305-6119

Xu, D., Zhang, J., Shi, Z., Song, C., Zheng, X., Zhang, Y., et al. (2015). Molecular detection of vector-borne agents in dogs from ten provinces of China. Parasit. Vectors 8:501. doi: 10.1186/s13071-015-1120-y

Yang, J., Li, Y., Liu, Z., Liu, J., Niu, Q., Ren, Q., et al. (2015a). Molecular detection and characterization of Anaplasma spp. in sheep and cattle from Xinjiang, northwest China. Parasit. Vectors 8:108. doi: 10.1186/s13071-0150727-3

Yang, J., Liu, Z., Niu, Q., Tian, Z., Liu, J., Guan, G., et al. (2015b). Tick-borne zoonotic pathogens in birds in Guangxi, Southwest China. Parasit. Vectors 8:637. doi: 10.1186/s13071-015-1249-8

Yaxue, Z., Hongtao, J., Qiuyue, W., Zhixin, F., Hongwei, G., Pengpeng, L., et al. (2011). Molecular detection of Anaplasma phagocytophilum in Ixodid ticks in Hebei Province, China. Vector Borne Zoonotic Dis. 11, 1323-1327. doi: 10.1089/ vbz.2010.0253

Yu, P. F., Niu, Q. L., Liu, Z. J., Yang, J. F., Chen, Z., Guan, G. Q., et al. (2016). Molecular epidemiological surveillance to assess emergence and re-emergence of tick-borne infections in tick samples from China evaluated by nested PCRs. Acta Trop. 158, 181-188. doi: 10.1016/j.actatropica.2016.02.027

Zamoto-Niikura, A., Morikawa, S., Hanaki, K. I., Holman, P. J., and Ishihara, C. (2016). Ixodes persulcatus ticks as a vector for Babesia microti U.S. lineage in Japan. Appl. Environ. Microbiol. 82, 6624-6632. doi: 10.1128/AEM. 02373-16

Zhang, X. C., Yang, Z. N., Lu, B., Ma, X. F., Zhang, C. X., and Xu, H. J. (2014). The composition and transmission of microbiome in hard tick, Ixodes persulcatus, during blood meal. Ticks Tick Borne Dis. 5, 864-870. doi: 10.1016/j.ttbdis.2014. 07.009

Zhang, Y., Lv, Y., Cui, Y., Wang, J., Cao, S., Jian, F., et al. (2016). First molecular evidence for the presence of Anaplasma DNA in milk from sheep and goats in China. Parasitol. Res. 115, 2789-2795. doi: 10.1007/s00436-016-5028-z

Zhao, X. G., Li, H., Sun, Y., Zhang, Y. Y., Jiang, J. F., Liu, W., et al. (2013). Dual infection with Anaplasma phagocytophilum and Babesia microti in a Rattus norvegicus, China. Ticks Tick Borne Dis. 4, 399-402. doi: 10.1016/j.ttbdis.2013. 04.002

Zhou, X., Xia, S., Huang, J. L., Tambo, E., Zhuge, H. X., and Zhou, X. N. (2014). Human babesiosis, an emerging tick-borne disease in the People's Republic of China. Parasit. Vectors 7:509. doi: 10.1186/s13071-014-0509-3

Conflict of Interest Statement: The authors declare that the research was conducted in the absence of any commercial or financial relationships that could be construed as a potential conflict of interest.

Copyright $(2016$ Wei, Song, Liu, Wang, Wang, Wang, Ma, Li, Zeng, Qian and Liu. This is an open-access article distributed under the terms of the Creative Commons Attribution License (CC BY). The use, distribution or reproduction in other forums is permitted, provided the original author(s) or licensor are credited and that the original publication in this journal is cited, in accordance with accepted academic practice. No use, distribution or reproduction is permitted which does not comply with these terms. 